\title{
On the Correct Convergence of Complex Langevin Simulations for Polynomial Actions *
}

\author{
H. Gausterer \\ Institut für Theoretische Physik, \\ Universität Graz, A-8010 Graz, AUSTRIA
}

September 1993

\begin{abstract}
There are problems in physics and particularly in field theory which are defined by complex valued weight functions $e^{-S}$ where $S$ is a polynomial action $S: \mathbb{R}^{n} \rightarrow \mathbb{C}$. The conditions under which a convergent complex Langevin calculation correctly simulates such integrals are discussed. All conditions on the process which are used to prove proper convergence are defined in the stationary limit.
\end{abstract}

*Supported by Fonds zur Förderung der Wissenschaftlichen Forschung in Österreich, project P7849. To appear in J. Phs. A. 


\section{Introduction}

Complex Langevin (CL) methods have turned out to be quite useful in the calculation (simulation) of high dimensional integrals over complex valued weight functions of the form $e^{-S}$, where $S$ is the action or the Hamiltonian of some physical system. Since there is no formal restriction to a real valued drift term for Langevin equations, the application of CL is convincingly simple [1]. Unfortunately one has to deal with two problems of uncertainty. The first is that it is apriori unknown whether the process will converge at all. The second problem is that, although the process has converged, it will not necessarily give the correct answer. This is, that long time averages of such a process do not necessarily simulate the complex valued weight function integrals. CL is known to sometimes give the wrong answer (see e.g. [2]).

Several attempts have been made to understand CL (e.g. see references [2, 3, ㄴ) . For some simple actions the behavior of CL can be improved by modifying the drift term with an appropriate kernel, but for general problems the choice of the kernel is not clear [5]. Recently progress has been made in the comprehension of the results which one gets from a convergent process [6, 7]. In particular the assumptions needed to guarantee correct results for convergent processes on certain compact manifolds $\left(S_{1}, S_{2}\right)$ turn out to be surprisingly simple and easy to verify in a numerical simulation. Contrary to that, many assumptions are used to prove the behavior of processes on $\mathbb{R}^{n}$ driven by polynomial actions and moreover these assumptions are rather technical [6].

For polynomial actions a lot of attention has been given to the existence of a pseudo Fokker-Planck (F-P) equation which describes the dynamics of a possibly equivalent complex valued weight function [6]. In earlier investigations especially the spectrum of this operator played a major role [1]. But statements on the properties of the spectrum are not sufficient to draw conclusions on the correctness or the convergence of CL [6]. Certainly, if one can show that the pseudo F-P equation exists and that the real part of the spectrum of the operator is semidefinite then CL converges but not necessarily to the desired result. Further conditions must hold (see [6]). Except for very simple cases it is hard and most unlikely to get exact information on the complete spectrum. Certainly there always exist the real F-P operator for the process and the convergence of the process follows if one can prove that the operator has a unique nonnegative integrable solution to the zero eigen- 
value. But this is also very hard and so far there is no classification scheme for actions which have the suitable properties. So, to get information on the convergence for any problem one must check either the existence and the whole spectrum of the pseudo F-P operator or the zero eigenvalue properties of the real F-P operator. In practice therefore the question of convergence still remains a matter of experiment and experience.

\section{Proper Convergence}

Let us now turn to the main purpose of the paper and examine in a rigorous fashion the conditions under which CL, if convergent, gives the right answer. To demonstrate this, several conditions at finite time have been put on the process in reference [6]. In this approach fewer conditions on the process are used and these conditions are put forward to $t \rightarrow \infty$. For simplicity the discussion and the formulas are restricted to the one dimensional case. All following statements allow for an immediate generalization to $\mathbb{R}^{n}$. It will be assumed that the system of interest is described by a complex polynomial action of degree $N$

$$
S(x)=\sum_{n=0}^{N} a_{n} x^{n} .
$$

$S: \mathbb{R} \rightarrow \mathbb{C}$ such that $e^{-S} \in \mathcal{S}(\mathbb{R}) . \mathcal{S}(\mathbb{R})$ is the Schwartz space of $C^{\infty}$ functions of rapid decrease. With $g(x)$ a polynomial of degree $M$ it is thus guaranteed, that the quantities of physical interest

$$
\begin{aligned}
\langle g(x)\rangle & \equiv \frac{1}{\mathcal{N}} \int_{\mathbb{R}} g(x) e^{-S(x)} d x, \\
\mathcal{N} & =\int_{\mathbb{R}} e^{-S(x)} d x
\end{aligned}
$$

do exist, provided $0<|\mathcal{N}|$. If $S$ would be real valued everything would be now straight forward ergodic theory and the longtime averages computed with the Langevin equation would reproduce the ensemble average of the system. [8])

In the complex case analytic continuation leads to the following stochastic differential equation.

$$
d Z(t)=F(Z(t)) d t+d W(t)
$$


with the drift term

$$
F(z)=-\frac{1}{2} \frac{d S(z)}{d z} .
$$

$W(t)$ is a standard Wiener process with zero mean and covariance

$$
E\left(W\left(t_{1}\right) W\left(t_{2}\right)\right)=\min \left(t_{1}, t_{2}\right) .
$$

Equation 2.4 is the so called CL equation. This equation has a locally unique solution which is defined up to a random explosion time [9]. In particular equation 2.4 describes a two dimensional diffusion process.

$$
\begin{gathered}
d X(\tau)=G(X(\tau), Y(\tau)) d \tau+d W(\tau), \\
d Y(\tau)=H(X(\tau), Y(\tau)) d \tau .
\end{gathered}
$$

With $S(z)=u(x, y)+i v(x, y)$, we have

$$
G(x, y)=-\frac{1}{2} \frac{\partial u(x, y)}{\partial x}, \quad H(x, y)=\frac{1}{2} \frac{\partial u(x, y)}{\partial y} .
$$

Special for this process is that equation 2.8 looks like a deterministic equation due to the zero diffusion coefficient. Nevertheless this is a stochastic equation through the dependence on $X(t)$. The singular diffusion matrix causes a lot of problems. So, contrary to the real action case it is in general not possible to determine from the drift and diffusion terms whether there exists a unique stationary distribution density for this process $[\mathbb{B}]$. As already mentioned in the introduction there is no general proof on the existence of a stationary distribution density. For the moment let us assume that for the process $X(t), Y(t)$ there exists a unique stationary distribution density $\hat{f}(x, y)$. The idea behind CL then is that

$$
\begin{aligned}
& \lim _{t \rightarrow \infty} E(g(X(t)+i Y(t)))= \\
& \int_{\mathbb{R}^{2}} g(x+i y) \hat{f}(x, y) d x d y=\frac{1}{\mathcal{N}} \int_{\mathbb{R}} g(x) e^{-S(x)} d x .
\end{aligned}
$$

might hold. 
Assume:

1. $S$ is a complex valued polynomial action of degree $N$ such that

$$
e^{-S} \in \mathcal{S}(\mathbb{R})
$$

and

$$
\left|\int_{\mathbb{R}} e^{-S(x)} d x\right|>0
$$

2. For

$$
c(k, t) \equiv E\left(e^{i k Z(t)}\right)=\int_{\mathbb{R}^{2}} e^{i k(x+i y)} f(x, y, t) d x d y
$$

the limit $t \rightarrow \infty$ exists pointwise and

$$
\lim _{\tau \rightarrow \infty} c_{\tau}(k) \equiv c_{\infty}(k) \in \mathcal{S}(\mathbb{R})
$$

3. Further

$$
\lim _{t \rightarrow \infty}\left|E\left(Z^{n}(t) e^{i k Z(t)}\right)\right|<\infty \text { for all } 0 \leq n \leq N-1, k \in \mathbb{R} .
$$

Equation 2.10 then holds at least for $g(z)$ a polynomial of degree $M \leq N-1$. Moreover equation 2.10 holds for any higher moment $E\left(Z^{n}(t)\right), n \geq N$ which exist for $t \rightarrow \infty$.

From assumption 2 we know that there is a $t_{0}<\infty$ such that $c(k, t)$ exists and from assumption 3 that there is a $t_{1}<\infty$ such that $E\left(Z^{n}(t) e^{i k Z(t)}\right)$ exists. Applying the Itô rule one gets with $F(z)$ as defined in 2.5

$$
\frac{\partial E\left(e^{i k Z(t)}\right)}{\partial t}=i k E\left(e^{i k Z(t)} F(Z(t))\right)-\frac{k^{2}}{2} E\left(e^{i k Z(t)}\right)
$$

Due to assumptions 2 and 3 equation 2.16 exists for $t^{\prime}=\max \left(t_{0}, t_{1}\right)$. As a side result we get that, if $c(k, t) \in C^{N-1}(\mathbb{R})$ with repsect to $k$, equation 2.16 can be understood as the dynamical equation for $c(k, t)$.

$$
\frac{\partial c(k, t)}{\partial t}=-\frac{i k}{2} \sum_{n=1}^{N} n a_{n}\left(-i \frac{\partial}{\partial k}\right)^{n-1} c(k, t)-\frac{k^{2}}{2} c(k, t) .
$$

Note that if assumption 3 does not hold, equation 2.16 can also not be defined in the sense of distributions. This is because we are not simply dealing with Fourier transforms but with their possibly not existing analytic continuations. 
Let us define now $\hat{h}(x)$ as

$$
\hat{h}(x)=\frac{1}{2 \pi} \int_{\mathbb{R}} c_{\infty}(k) e^{-i k x} d k
$$

From assumption 2 follows that $\hat{h}(x) \in \mathcal{S}(\mathbb{R})$. Using equation 2.18 and assumption 3

$$
\lim _{t \rightarrow \infty} E\left(Z^{n}(t) e^{i k Z(t)}\right)=\int_{\mathbb{R}} x^{n} e^{i k x} \hat{h}(x) d x
$$

for $0 \leq n \leq N-1$ and $k \in \mathbb{R}$. Applying the above result to 2.16 one obtains in the limit $t \rightarrow \infty$

$$
0=i k \int_{\mathbb{R}} e^{i k x} F(x) \hat{h}(x) d x-\frac{k^{2}}{2} \int_{\mathbb{R}} e^{i k x} \hat{h}(x) d x
$$

Integrating the right hand side of equation 2.20 by parts gives that $\hat{h}(x)$ is a $L^{1}(\mathbb{R}, d x)$ zero eigenvalue solution of a F-P type differential operator with a complex drift term (pseudo F-P operator).

$$
\frac{1}{2} \frac{\partial}{\partial x}\left[\frac{\partial S(x)}{\partial x}+\frac{\partial}{\partial x}\right] \hat{h}(x) \equiv \mathcal{T} \hat{h}(x)=0 .
$$

$\mathcal{T}$ has two zero eigenvalue solutions. One is

$$
\hat{h}_{1}(x) \sim e^{-S(x)} \in \mathcal{S}(\mathbb{R})
$$

which fits to assumption 2, since as the Fourier transform of $c_{\infty}(k)$ it must be a Schwartz function. For the second solution

$$
\hat{h}_{2}(x) \sim e^{-S(x)} \int_{x_{0}}^{x} e^{S(y)} d y
$$

one can show that

$$
\hat{h}_{2}(x)=\mathcal{O}\left(\frac{1}{x^{N-1}}\right) \text { for }|x| \rightarrow \infty
$$

This contradicts assumption 2. So, the only possible solution is the one proportional to $e^{-S}$ and

$$
\lim _{t \rightarrow \infty} E\left(Z^{n}(t) e^{i k Z(t)}\right)=\frac{1}{\mathcal{N}} \int_{\mathbb{R}} x^{n} e^{i k x} e^{-S(x)} d x
$$


for $0 \leq n \leq N-1$ and $k \in \mathbb{R}$. If further $E\left(Z^{n}(t)\right), n \geq N$ for $t \rightarrow \infty$ exist then

$$
\lim _{t \rightarrow \infty} E\left(Z^{n}(t)\right)=\left.\frac{d^{n} c_{\infty}(k)}{d k^{n}}\right|_{k=0}=\frac{1}{\mathcal{N}} \int_{\mathbb{R}} x^{n} e^{-S(x)} d x .
$$

Let us now briefly discuss the assumptions. Polynomial actions are very natural since most physical systems defined on $\mathbb{R}^{n}$ have polynomial actions. Since these actions must be bounded from below it follows that $e^{-S} \in \mathcal{S}$. Condition 2 must be there otherwise the solution $\hat{h}_{2}(x)$ cannot be excluded. With the correctness requirement on CL that

$$
\lim _{t \rightarrow \infty} E\left(e^{i k Z(t)}\right)=\frac{1}{\mathcal{N}} \int_{\mathbb{R}} e^{i k x} e^{-S(x)} d x
$$

this condition is also a necessary condition. Assumption 3 looks technical, but is so far required to relate $\hat{h}_{i}(x)$, the Fourier transform of $c_{\infty}(k)$, to the Fokker-Planck type operator $\mathcal{T}$. This condition finally allows to show the correctness of CL. It would be nice to eliminate assumption 3 by showing that it follows from assumption 2. Unfortunately the integral transform defined by equation 2.13 is not an injective mapping. To the authors knowledge the nature of this integral transform has not been analized in the literature. At present, without more detailed information on the probability density (in general not available), it is perhaps impossible to draw conclusion on the properties of the function from the properties of its image. In a numerical simulation certainly such mathematical criteria a hard to verify exactly. Nevertheless experience tells us that when plotting such expectation values $\left(c_{\infty}(k), E\left(Z^{n} e^{i k Z}\right)\right)$ one gets a very clear sign of the quality of the result [10].

\section{Conclusions}

The criteria under which a convergent CL simulation leads to correct results have been significantly simplified. The assumptions used in the present proof are much closer to a numerical verification than the one used in reference [6]. Unfortunately a complete theory of CL is still lacking. However the situation that it was generally neither apriori nor aposteriori possible to prove convergence to the desired result has been ameliorated in as far as a simple aposteriori proof is now possible. 


\section{References}

[1] J.R. Klauder, in: Recent Developments in High Energy Physics, eds. H. Mitter and C.B. Lang (Springer, Wien, New York, 1983)

[2] J.R. Klauder, W.P. Petersen, J. Stat. Phys. 39 (1985) 53

[3] J. Ambjorn, S. K. Yang, Phys. Lett. B 165 (1985) 140;

[4] R.W. Haymaker, Y. Peng, Phys. Rev. D 41 (1990) 1269;

[5] H. Okamoto, K. Okano, L. Schülke, S. Tanaka, Nucl. Phys. B 324 (1989) 684; K. Okano, L. Schülke, B. Zheng, Phys. Lett. B 258 (1991) 421;

[6] H. Gausterer, S. Lee, UNIGRAZ-UTP 29-09-92, to be published in J. Stat. Phys. 73 (1993)

[7] S. Lee, to be published in Nucl. Phys. B

[8] H. Risken, The Fokker-Planck Equation (Springer, Berlin 1984); C.W. Gardiner, Handbook of Stochastic Methods (Springer, Berlin 1983)

[9] L. Arnold, Stochastic Differential Equations ( Wiley, New York 1974)

[10] S. Lee, University of Florida Gainesville Thesis (1993) 